\title{
Molecular dynamics simulations of thermal evaporation and critical electric field of copper nanotips
}

\section{Gao, Xinyu}

2020-09-02

Gao , X, Kyritsakis , A, Veske, M, Sun , W , Xiao, B, Meng, G, Cheng , Y \& Djurabekova , F 2020 , ' Molecular dynamics simulations of thermal evaporation and critical electric field of copper nanotips ' , Journal of Physics. D, Applied Physics , vol. 53 , no. 36 , 365202 . https://doi.org/10.1088/1361-6463/ab9137

http://hdl.handle.net/10138/338810

https://doi.org/10.1088/1361-6463/ab9137

cc_by_nc_nd

acceptedVersion

Downloaded from Helda, University of Helsinki institutional repository.

This is an electronic reprint of the original article.

This reprint may differ from the original in pagination and typographic detail.

Please cite the original version. 


\title{
Molecular dynamics simulations of thermal evaporation and critical electric field of copper nanotips
}

\author{
Xinyu Gao ${ }^{1}$, Andreas Kyritsakis ${ }^{2}$, Mihkel Veske ${ }^{2}$, Wenjie Sun ${ }^{1}$, Bing Xiao ${ }^{1 *}$, \\ Guodong Meng ${ }^{1}$, Yonghong Cheng ${ }^{{ }^{*}}$ and Flyura Djurabekova ${ }^{{ }^{*}}$ \\ ${ }^{1}$ State Key Laboratory of Electrical Insulation and Power Equipment, Xi'an Jiaotong University, Xi'an \\ 710049, People's Republic of China \\ ${ }^{2}$ Helsinki Institute of Physics and Department of Physics, University of Helsinki, PO Box 43, FI-00014 \\ Helsinki, Finland
}

E-mail: bingxiao84@xjtu.edu.cn,cyh@mail.xjtu.edu.cn, flyura.djurabekova@helsinki.fi

\begin{abstract}
Due to the miniaturization of microelectromechanical systems (MEMS), nanoelectromechanical systems (NEMS) and molecular devices, the problem of vacuum insulation becomes more and more prominent. The nanoscale thermal effects caused by electron emission and electric current Joule heat under high electric field lead to gasification and migration of material in the device. In this work, a coupled molecular dynamics electrodynamics method is used to simulate the thermal evaporation of nanotips under high electric field. Moreover, $\mathrm{Cu}$ nanotips with different initial geometries and different macroscopic electric fields are modelled. The deformation and damage mechanisms of nanotips under high electric field are discussed. Our simulations show that the aspect ratio of nanotips has a significant influence on the thermal evaporation of nanotips. The thermal runaway occurring in picosecond time-scale plays an important role for the initiation of the vacuum breakdown. An empirical relationship is obtained between the on-set breakdown time and the macroscopic electric field and the geometry of nanotips by analysing the numerical results.
\end{abstract}

Keywords: vacuum discharges, field emission, high electric fields, molecular dynamics

\section{Introduction}

Vacuum discharges, as well as their significant applications and undesirable destructiveness, has become research hotspots both in plasma technology and electrical insulation. For example, particle accelerators like Compact electron-positron Linear Collider (CLIC) [1] designed and operated by the European Organization for Nuclear Research (CERN), its internal electrical insulation structure needs to withstand extremely high field strength of at least $100 \mathrm{MV} / \mathrm{m}$ [2]. In micro- or nanoelectronic devices operating under high electric fields, significant field emission currents may appear. These can lead directly to electrical breakdowns, which will eventually cause the complete destruction of the entire electronic component. As the miniaturization of micro- or nanoelectronic devices such as nanoelectromechanical systems (NEMS), the problem of vacuum insulation in the devices under high electric fields becomes more and more prominent [3]. On the other hand, vacuum discharges also play a vital role in arc jet, ion sources [4] and fusion reactors [5] in the field of aerospace or nuclear physics research.

After extensive research since 1950s [6 15], it was realized that the physical law of macro-scale can no longer be applied to explain the development of the vacuum discharge because of the new physical effects brought by the decrease of dimension down to the micro- or nanoscale. An explosive emission mechanism was proposed by Mesyats [16, 17], which could be used to interpreted the initiation of vacuum breakdown in RF accelerating structures [18 20]. Recent studies on micro/nano scales breakdown characteristics showed that field electron emission or thermionic emission play a significant role in breakdown process [21 29]. Nanoscale breakdown characteristics have been investigated based on nanometer displacement platform [30 32] and it was 
found that when the local field strength is greater than 350 $\mathrm{MV} / \mathrm{m}$, field electron emission was generated on the surface of the cathode, and which caused the rise of the temperature of field emitter and anode [32]. The resulting metal vapor, would collide with electrons to form plasma, leading to electrical breakdown. Recent simulation results showed the effects of vacuum nano-gap and cathode materials on the dielectric strength of the vacuum nano-gap of the planar electrode [33]. In addition, series of electrodynamicsmolecular dynamics simulations were conducted to understand a thermal runaway process on nano-tip emitters under high electric field [34 37]. It was shown that this process leads to the evaporation of the electrode tip as the electric field-induced force elongates and sharpens it [38].

Our previous experiments [39 41] further showed that there is a correlation between nano-scale vacuum breakdown characteristics and the properties specific to the electrode material. Moreover, a significant role may play the geometry of electrodes and the gap length $[42,43]$. However, no clear physical mechanism has been yet established to explain the correlation between the electric breakdown and the properties of the electrodes.

In this work, we use the coupled molecular dynamics (MD) - electrodynamics (ED) method of [38] to investigate the dependence of the thermal runaway process of $\mathrm{Cu}$ nanotips with respect to its geometrical characteristics. The results could provide the vital information to further understand the fundamental mechanism of vacuum breakdown at nanoscale.

\section{Methods}

In our previous experimental works, we employed a custom SEM-FIB experiment system to study the vacuum discharge in the nano-size gap between two nano-electrodes, as shown in figures 1(a) and (b) [39 43]. However, current experimental methods are unable to capture the exact moment of the initiation of vacuum discharge and the associated structural evolution dynamics of electrodes due to the constraints of experimental apparatus down to the nano-scale in size and to ps in time-scale. Meanwhile, the traditional classical molecular dynamics simulation method is not suitable to simulate the structural evolution of nano-electrodes under high electric field, because the effects of high electric field at the macroscopic scale on the microscopic structure of nano-electrode are not properly described or implemented in the methodology. The electron emission [44 46], the Joule effect, the Nottingham effect [47, 48] and the resulting structural changes under high electric field are supposed to play important role in vacuum electric breakdown. In this work, a very recently developed FEMOCS code [36 38], which combined the classical MD (PARCAS [49 51]) with electrodynamics, was used to simulate the thermal evaporation of nanotips under high electric field. In the EDMD simulation, the structural evolution at atomic scale is

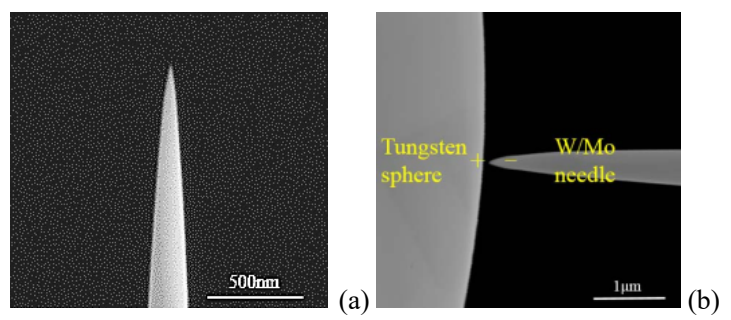

Figure 1. Experimental method of nanoscale vacuum discharge: (a) metal nanometer electrodes fabricated by a SEM-FIB experiment system; (b) SEM image of nanoscale vacuum discharge system with nano-gap formed by nanomanipulators.

governed by the Newton's equation of motion. Meanwhile, the mixed Neumann and Dirichlet boundary conditions are used to obtain the electrostatic potential and electric field in the vacuum region and on the surface of electrodes by solving the Lapace equation. Then the effects of the forces induced on the material surface by the electrostatic field are dynamically incorporated into the MD simulation.

\subsection{Calculation method of physical process}

In this work, all the calculations were based on FEMOCS $[36,38]$. The electric field distribution was calculated by solving the Laplace equation on the flexible finite element method (FEM) [52] mesh

$$
\nabla^{2} \phi=0 .
$$

We applied a Dirichlet boundary condition on the surface of metal electrode:

$$
\phi=0,
$$

and a Neumann boundary condition on the upper surface of the simulation box:

$$
\nabla \phi=\hat{z} E_{0},
$$

where $E_{0}$ is the applied macroscopic electric field at the top of the FEM mesh. By calculating the induced charges $q_{i}$ on the surface atoms, we obtained the resulting Lorentz and Coulomb forces by $[34,38]$

$$
\overrightarrow{F_{i}}=\frac{1}{2} q_{i} \vec{E}_{i}+\frac{1}{4 \pi \varepsilon_{0}} \sum_{j=1}^{N_{\text {neig }}} \frac{q_{i} q_{j}}{r_{i j}^{2}} e^{-r_{i j} \xi} \hat{r}_{i j} .
$$

The electron emission current [53 56] and Nottingham heat conduction from the tip were calculated by GETELEC code [57] integrated in FEMOCS, which can automatically distinguishes between different emission conditions over the barrier based on the Kemble formula. In addition, the overall heat diffusion equation is given by [58]

$$
C_{V} \frac{\partial T}{\partial t}=\nabla \cdot(\kappa(T) \nabla T)+p
$$

where $C_{V}$ is the isothermal heat capacity, $\kappa(T)$ is the thermal conductivity and $p$ is the local deposited volumetric heating power density (in $\mathrm{W} \mathrm{m}^{-3}$ ). 
Table 1. The structural and modeling parameters (Number of atoms, MD box size and FEM mesh box size) of different copper nanotips.

\begin{tabular}{ccccc}
\hline$R_{0}(\mathrm{~nm})$ & $H_{0}(\mathrm{~nm})$ & Number of atoms & MD box size $\left(\mathrm{nm}^{3}\right)$ & FEM mesh box size $\left(\mathrm{nm}^{3}\right)$ \\
\hline 1 & 100 & 78658 & $7.14 \times 7.14 \times 51.18$ & $695.91 \times 695.91 \times 1004.91$ \\
3 & 80 & 104451 & $9.09 \times 9.09 \times 30.18$ & $677.14 \times 677.14 \times 808.72$ \\
3 & 100 & 233970 & $11.27 \times 11.27 \times 50.17$ & $685.81 \times 685.81 \times 1008.72$ \\
3 & 120 & 423981 & $13.09 \times 13.09 \times 70.17$ & $680.21 \times 680.21 \times 1208.65$ \\
5 & 100 & 482514 & $14.91 \times 14.91 \times 50.17$ & $677.32 \times 677.32 \times 1008.74$ \\
\hline
\end{tabular}

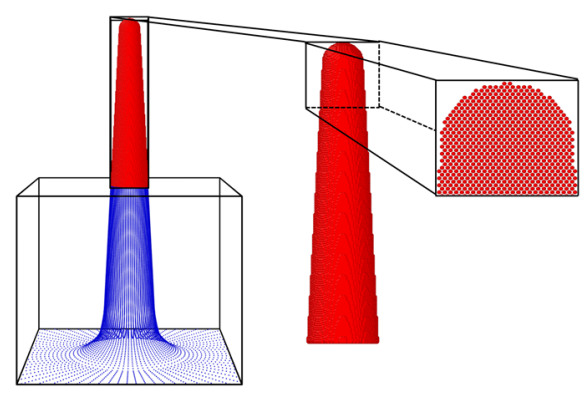

(a)

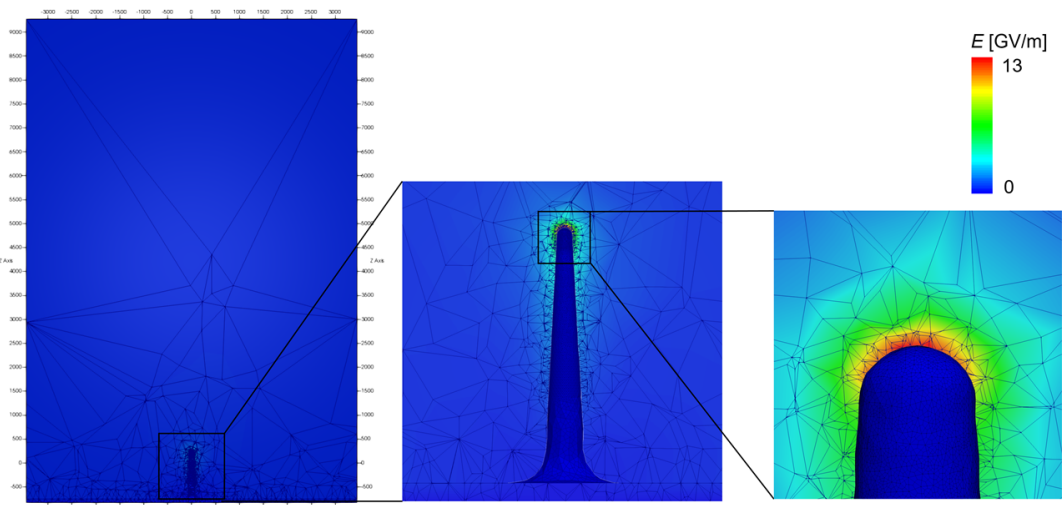

Figure 2. (a) Initial modeling geometry of $\mathrm{Cu}$ nanotip consisting of the small 'real' electrode tip region and the large extended 'virtual' stand. (b) Profiles of the FEM mesh with different mesh densities at nanotip apex, lateral facet and substrate. The color coding represents the distribution of the electric field.

A Neumann boundary condition was added to the heat equation to calculate Nottingham heat $\left(p_{N}\right)$ [38]:

$$
\hat{n} \cdot(\kappa \nabla T)=p_{N} .
$$

The details about calculation of $p$ and $p_{N}$ can be found from [38] and [57]. In addition, a Berendsen temperature controlling scheme [59] with time constant $\tau=1.5 \mathrm{ps}$ was used to scale the velocities of the atoms at each MD iteration.

\subsection{Modeling method of MD simulation}

Our simulation geometry was based on the previous experimental investigation [41] and theoretical simulation [38]. $\mathrm{Cu}$ nanotips (major axis aligned with the $<100>$ crystallographic direction) with different initial geometries (the radius of the tip hemisphere $\left(R_{0}\right)$ and total tip height $\left(H_{0}\right)$ ) and different macroscopic electric fields (100 MV/m 1 GV/m) were modeled, as shown in figure 2 (a). For all nanotips, the taper angle was fixed to $3^{\circ}$.

To achieve higher efficiency for the ED-MD simulations, the whole nano-electrode includes the tip region (small rectangular box filled with atoms in red) and extended stand (large cubic box filled with blue dots). As can be seen from figure 1, the tip of the electrode was occupied with the actual atoms which have the same position registry to that of bulk FCC copper. The tip region was subjected to the molecular dynamics simulation in which the coordinate and temperature of the last two bottom atomic layers were fixed. The
Sabochick and Lam (SL) EAM potentials [60] were used for the MD simulations with a constant time step $\Delta t=4.05 \mathrm{fs}$ and the initial temperature $T_{0}=300 \mathrm{~K}$. Meanwhile, the extended stand in the lower large box was built with the 'virtual' blue support points [38]. All those blue points were placed on the surface of the copper electrode, which defined the boundary between metal electrode and vacuum. The extended region was used to generate the flexible FEM mesh (shown in figure 2(b)) for obtaining the numerical solutions of electrostatic and heat transfer equations. In the simulation, the height of large box for the extended stand was set to $50 \mathrm{~nm}$, while the height of the nanotip was defined by the initial geometry of electrode as given in table 1. Other detailed modelling parameters are also shown in table 1 .

Solving the Laplace equation, we could calculate the Field Enhancement Factor $(\gamma)$ by

$$
\gamma=\frac{E_{\text {apex }}}{E_{0}}
$$

where $E_{\text {apex }}$ is the electric field at the apex of the nanotips. Fitting the relation between $\gamma$ and aspect ratio $\left(H_{0} / R_{0}\right)$ of nanotips by expression in [61], we obtained

$$
\gamma=1.38 \times\left(4.2+\frac{H_{0}}{R_{0}}\right)^{0.81}
$$

The maximum deviation of $\gamma$ values in our simulations from the results in [61 65] is less than $24 \%$, which may be because our tips have a certain taper. 


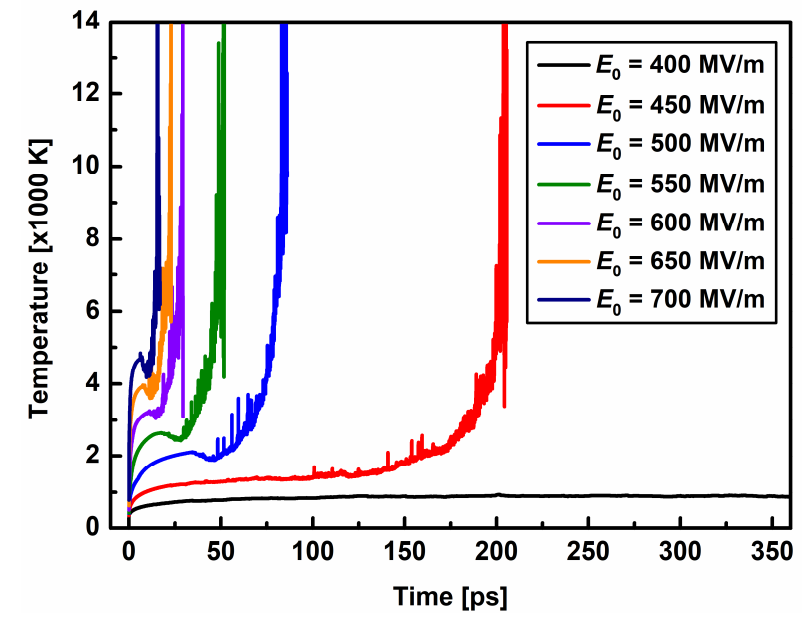

Figure 3. The time evolution of the maximum temperature on the tip with different macroscopic field strengths from $400 \mathrm{MV} / \mathrm{m}$ to $700 \mathrm{MV} / \mathrm{m}$.

\section{Results and discussions}

\subsection{Effects of field strength on the thermal field of nanotips}

We show the time evolution of the maximum temperature $\left(T_{\max }\right)$ on the tip under different electric fields for the nanotip of $R_{0}=3 \mathrm{~nm}$ and $H_{0}=100 \mathrm{~nm}$ in figure 3. It is apparent that $T_{\max }$ has significant influence on electric field $\left(E_{0}\right)$. On the one hand, the maximum temperature on the tip tends to be stabilized in the range from $800 \mathrm{~K}$ up to $1000 \mathrm{~K}$ when the macroscopic fields $E_{0}=400 \mathrm{MV} / \mathrm{m}$. In contrast, when $E_{0}$ is larger than $400 \mathrm{MV} / \mathrm{m}$, the maximum temperature on the tip can rapidly increase over $10000 \mathrm{~K}$. The stronger the macroscopic electric field, the less time it takes for this phenomenon to happen and the higher the temperature of the tip for the same MD run duration. Since the maximum local temperature on the tip is much higher than that of the melting point of bulk copper crystal, the crystal structure of nanotip is in the plasma state [16 18]. This drastic change of temperature not only leads to the great change of the geometry of nanotip but also increases electrical resistivity of the metal nanotip. The increasing of electrical resistivity gives the positive feedback to the Joule heat in the nanostructure and which in turn affects the tip temperature and electron emission in a thermal runaway process, as shown in [38]. Eventually, the high local temperature on the nanotip may cause atoms at the apex region to evaporate.

The time evolution of the Nottingham and Joule heats deposited on the $\mathrm{Cu}$ nanotip under different electric field are displayed in figure 4 (a) and (b), respectively. Similar to the trend of temperature evolution, there is no significant change in either Nottingham heat or Joule heat when $E_{0} \leq 400 \mathrm{MV} / \mathrm{m}$. For $E_{0}>400 \mathrm{MV} / \mathrm{m}$, the initial heating of the nanotip is due to the Nottingham effect caused by the field emission. As the

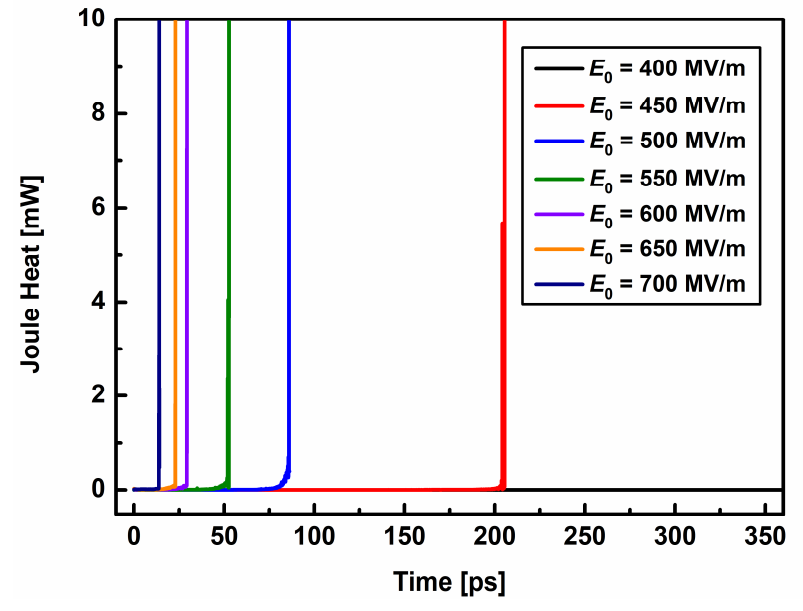

(a)

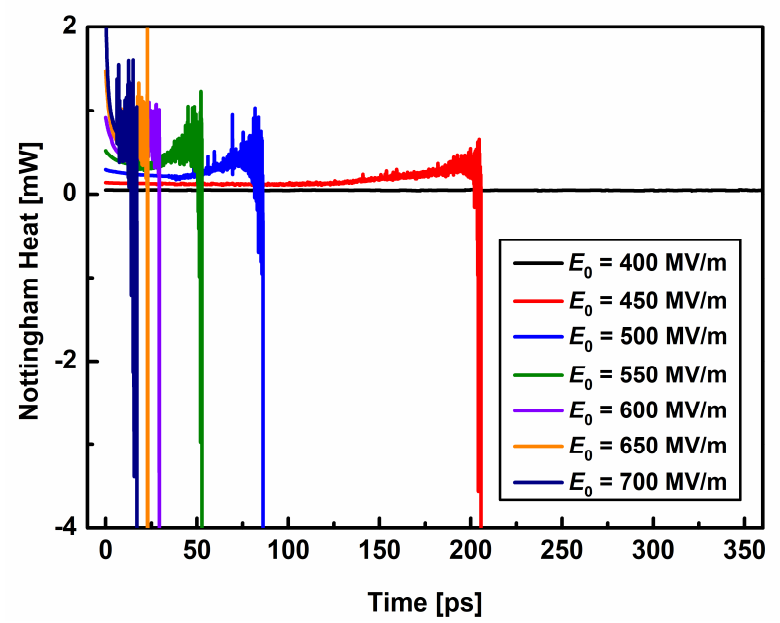

(b)

Figure 4. The time evolution of the Joule heat (a) and the Nottingham heat (b) deposited on the whole $\mathrm{Cu}$ nanotip with different macroscopic field strength from $400 \mathrm{MV} / \mathrm{m}$ to $700 \mathrm{MV} / \mathrm{m}$.

local temperature of nanotip exceeds $1000 \mathrm{~K}$, thermionic component of the emission will be added automatically by GETELEC [57]. Both Joule and Nottingham heats are positive at the beginning of the ED-MD simulation. The sign of Nottingham heat is inverted after a while with the significant increase of current density and temperature and which is resulted from the changing of electron emission mechanism from the cold field electron emission to thermionic emission on the nanotip. Meanwhile, the Joule heat remains positive and its value increases rapidly due to the increased thermionic emission. High electric fields induce the higher local temperature on nanotips, giving stronger thermionic emission. Consequently, the inversion of Nottingham heat occurs for a very short MD duration. Although the cooling effect of Nottingham cooling causes a delay in the heating process of the nanotip, the significant increase of the resistivity caused by the structural deformation or elongation at high temperature eventually leads to the dominance of Joule heat in the heat conduction. The structural evolution of nanotip under high electric field will be covered in the next session. 


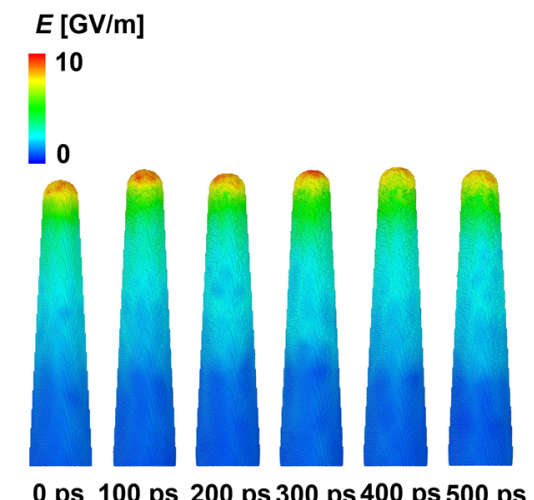

0 ps 100 ps 200 ps 300 ps 400 ps 500 ps

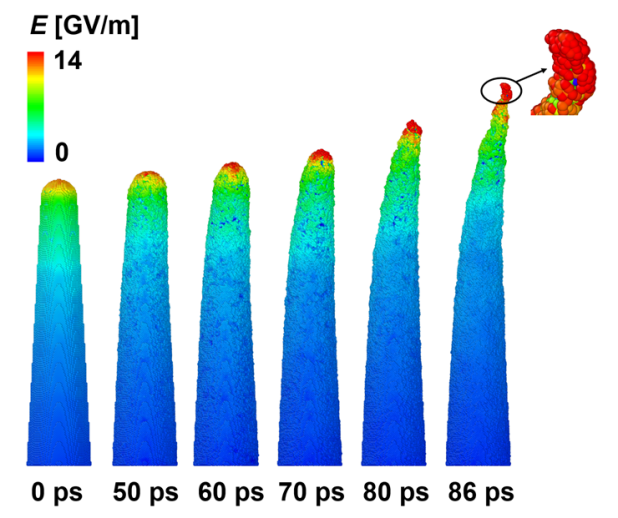

(a)

(b)

Figure 5. The evolution of the shape and surface electric field of nanotips $\left(R_{0}=3 \mathrm{~nm}, H_{0}=100 \mathrm{~nm}\right) \mathrm{when} E_{0}=400 \mathrm{MV} / \mathrm{m}(\mathrm{a})$ and $500 \mathrm{MV} / \mathrm{m}(\mathrm{b})$.

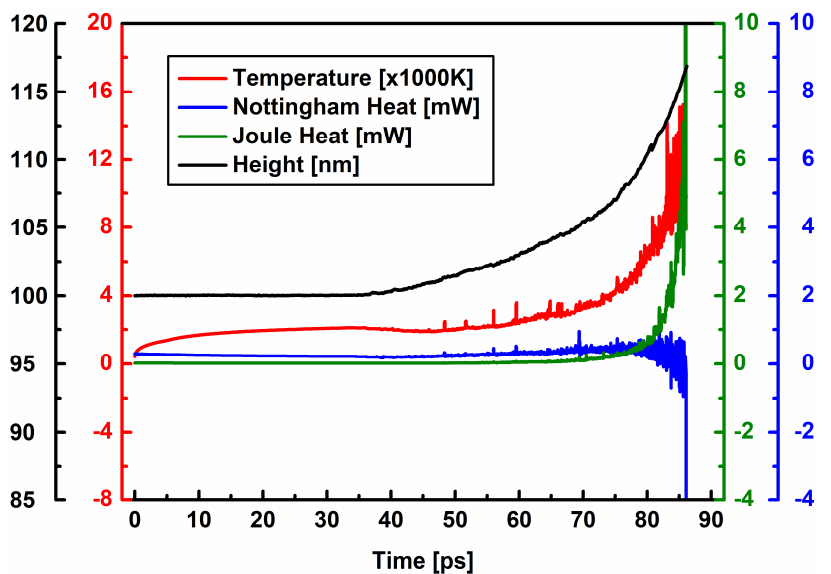

Figure 6. The time evolution of the height of the nanotip, maximum temperature on the tip, Joule and Nottingham heats deposited on the $\mathrm{Cu}$ $\operatorname{nanotip}\left(E_{0}=500 \mathrm{MV} / \mathrm{m}, R_{0}=3 \mathrm{~nm}\right.$ and $\left.H_{0}=100 \mathrm{~nm}\right)$.

\subsection{Effects of field strength on the height of nanotips}

From figure 3 and 4, it can be seen that the strength of the macroscopic electric field determines whether the tip tends to be stable or deform. We pick several snapshots in ED-MD simulations to illustrate the evolution of the shape and surface electric field of $\mathrm{Cu}$ nanotips $\left(R_{0}=3 \mathrm{~nm}, H_{0}=100 \mathrm{~nm}\right)$ when $E_{0}$ $=400 \mathrm{MV} / \mathrm{m}$ and $500 \mathrm{MV} / \mathrm{m}$ in figures $5(\mathrm{a})$ and (b), respectively. For $E_{0}=400 \mathrm{MV} / \mathrm{m}$ (figure 5(a)), the height of the nanotip varies in the range from $99 \mathrm{~nm}$ to $101 \mathrm{~nm}$ in the entire 500 ps MD run. In this case, the nanotip is stable against to the stress caused by the electric field and the rising of local temperature due to the deposition of Joule and Nottingham heat. The height of nanotip nearly remains a constant during the whole MD duration.

For $E_{0}=500 \mathrm{MV} / \mathrm{m}$, as shown in figure 5(b) and figure 6 $(\mathrm{t}<15 \mathrm{ps})$, the heat generated by the strong electron emission heats system at the apex region of the nanotip to exceed the melting point of $\mathrm{Cu}$. Later on $(\mathrm{t}<40 \mathrm{ps})$, the maximum temperature on the tip, Joule and Nottingham heats go into a steady state and their values fluctuate around mean values. When $\mathrm{t}>40 \mathrm{ps}$, the local electric field of nanotip reaches the critical value and the temperature at the apex starts rising.

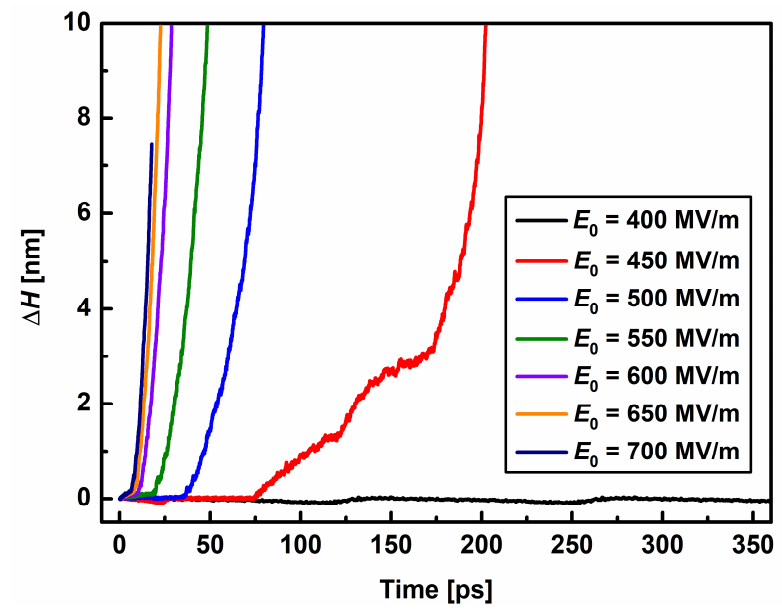

Figure 7. The time evolution of the height of nanotips under different electric fields

Under the simultaneous action of electrical and thermal stresses, the melting and subsequent migration of the atoms toward the anode continuously stretch the tip, increasing its height. The characteristic sharpening of tip is demonstrated in figure 5 (b) at this stage, resulting in the fast increasing of local electric field strength. The increasing of the tip height in the combination with the great increasing the resistivity hinder the heat dissipation, causing strong over heating at the nanotip. The sharpening of nanotip due to the high local temperature feeds positively back into the electric field and tip height, eventually resulting with the thermal runaway. After $\mathrm{t}>86 \mathrm{ps}$, the height of the nanotip is elongated up to $117 \mathrm{~nm}$, and the significant atomic evaporation can be observed at the apex region of the tip. This trend is consistent with the results in [38]. Figure 7 demonstrates the change of the height of nanotips $\left(\Delta H=H e i g h t(t)-H_{0}\right)$ under different electric fields. As it can be seen from figure 7 , both the rate of increase and the amplitude of $\Delta H$ get large by increasing the electric field strength. Interestingly, the calculated $\Delta H$ does not vary with the time when the macroscopic electric field is below certain value, i.e., $E_{0}<450 \mathrm{MV} / \mathrm{m}$ in the case discussed here. Therefore, the existence of the critical electric field strength in the process of atomic evaporation might be defined. 

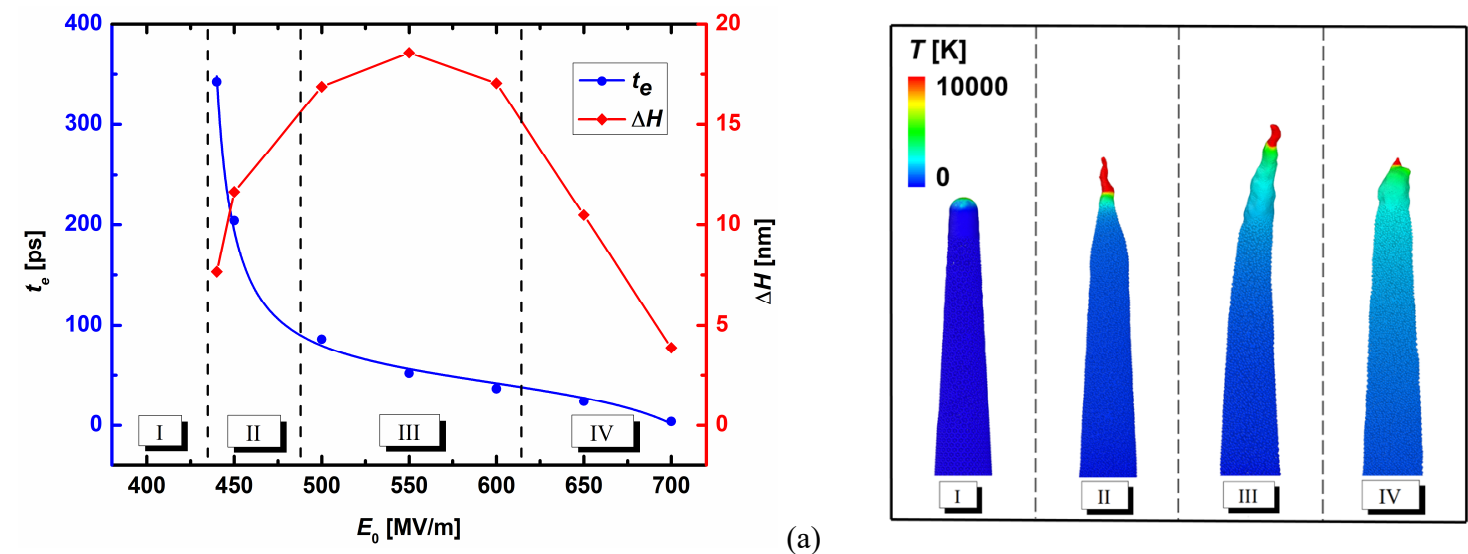

(a)

(b)

Figure 8. (a) The diagram of the initial time of thermal evaporation of atoms $\left(t_{e}\right)$ and $\Delta H$ under different $E_{0}$. (b) Morphologies of nanotips at various stages. The color coding of (b) corresponds to the temperature distribution of tips.

Table 2. The macroscopic electric field and the predicted onset time for the pre-breakdown for the nanotip of $R_{0}=3 \mathrm{~nm}$ and $H_{0}=100 \mathrm{~nm}$ ) using ED-MD simulation.

\begin{tabular}{lr}
\hline$E_{0}(\mathrm{MV} / \mathrm{m})$ & $t_{1}(\mathrm{ps})$ \\
\hline 440 & 342.18 \\
450 & 204.08 \\
500 & 86.18 \\
550 & 51.80 \\
600 & 36.60 \\
650 & 23.69 \\
700 & 14.17 \\
\hline
\end{tabular}

\subsection{Determining the onset time of thermal evaporation and the critical field strength}

The onset time of thermal evaporation and the corresponding electric field strength play the key role to define the pre-breakdown moment for the nanotips in the vacuum.

Based on a cluster analyzing algorithm [66] previously implemented in FEMOCS code and by using the cut-off radius of $4.94 \AA$, we define the onset time $\left(t_{1}\right)$ as the moment when a single atom or cluster is detached from the nanotip $\left(R_{0}=3 \mathrm{~nm}\right.$, $\left.H_{0}=100 \mathrm{~nm}\right)$. The predicted values under different electric fields are given in table 2 . We find that when $E_{0}=430 \mathrm{MV} / \mathrm{m}$, no atom is detached from the nanotip within $1 \mathrm{~ns}$ of simulation run. On the other hand, when $E_{0}=500 \mathrm{MV} / \mathrm{m}$, atoms start evaporating at $t_{1}=86.18 \mathrm{ps}$. For this particular ED-MD simulation $\left(E_{0}=500 \mathrm{MV} / \mathrm{m}\right)$, combining the results presented in figures 3, 4 and 6 , it can be found that the height, the maximum temperature of the tip, the Nottingham and Joule heats all change or increase abruptly at time $t_{l}$. In this case, we could define it as the onset time of thermal evaporation of atoms and the pre-breakdown moment for the nanotips.

After we repeated the ED-MD simulations for three times and measured $t_{1}$ for nanotips under various long-range electric fields, the mean pre-breakdown onset time $\left(t_{\mathrm{e}}\right)$ is obtained. The obtained values of $t_{\mathrm{e}}$ are shown in figure 8 . We should clarify that only the initial thermal runaway in pre-breakdown process is recorded during this time period, rather than a complete breakdown process as the explosive emission mechanism demonstrated including the expansion of plasma into vacuum gap. It is found that the value of $t_{\mathrm{e}}$ tends to infinity when $E_{0}=430 \mathrm{MV} / \mathrm{m}$ and thermal evaporation occurs when $E_{0}>430 \mathrm{MV} / \mathrm{m}$. Thus the critical field strength of the atomic thermal evaporation $\left(E_{c}\right)$ could be determined to be $430 \mathrm{MV} / \mathrm{m}$.

In figure 8 , we show the profiles of te and $\Delta H$ under different $E_{0}$. Four different regions are recognized in the profiles. In the Region I, the macroscopic field is less than the critical value. The effects of electric field are not strong enough to trigger the overheating of nanotip. The thermal evaporation does not occur, so that the morphology of tips does not change significantly. In Region II, the macroscopic electric field strength is slightly above the critical value. However, the electric field is still not sufficiently high to initiate the thermal evaporation in a very short time scale. The onset time decreases rapidly with the increasing of the macroscopic electric field strength, accompanying with the fast elongating and sharpening of nanotip structure. Region III is characterized by a slow varying of both parameters $t_{e}$ and $\Delta H$. In this region, the sharpening of the nanotip and the associated thermal evaporation occur throughout the entire tip, and the tip could be elongated to the extreme large value within relatively short time period. In the Region IV, due to the extremely high field strength, the overheating of the nanotip is severe especially at the apex region. The very high local temperature causes the intense thermal evaporation of the atoms on the nanotip. In this case, the nanotip melts rapidly in just a few picoseconds rather than being elongated gradually. The macroscopic electric field, that causes $t_{e}=3 \mathrm{ps}$, is defined as $E_{\max }$ in this work. The onset time for the thermal evaporation or pre-breakdown is considered to be infinitesimally small for $E>E_{\max }$. 

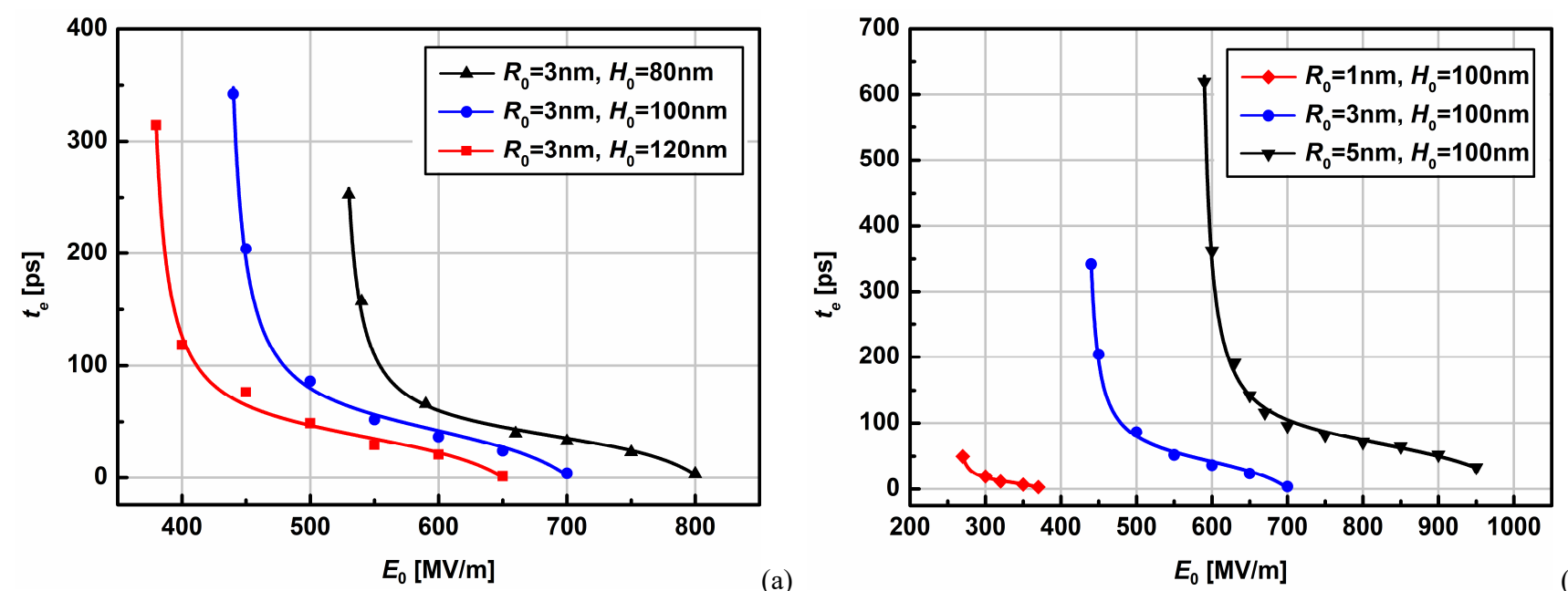

Figure 9. The diagram of the initial time of thermal evaporation of nanotips with different $H_{0}(80 \mathrm{~nm}, 100 \mathrm{~nm}$ and $120 \mathrm{~nm})$ and $R_{0}(1 \mathrm{~nm}, 3 \mathrm{~nm}$ and $5 \mathrm{~nm})$ under various electric fields.

Table 3. The values of fitting parameters with different $R_{0}$ and $H_{0}$.

\begin{tabular}{ccccccc}
\hline$R_{0}(\mathrm{~nm})$ & $H_{0}(\mathrm{~nm})$ & $E_{c}(\mathrm{MV} / \mathrm{m})$ & $E_{\max }(\mathrm{MV} / \mathrm{m})$ & $\tau$ & $t_{h}$ & $R M S E\left(t_{e}\right)$ \\
\hline 5 & 100 & 580 & 1075 & 35.63 & 67.52 & 9.85 \\
3 & 80 & 520 & 856 & 20.65 & 37.56 & 4.80 \\
3 & 100 & 430 & 765 & 28.70 & 42.56 & 5.88 \\
3 & 120 & 370 & 717 & 25.11 & 36.36 & 5.85 \\
1 & 100 & 260 & 411 & 8.42 & 10.10 & 1.02 \\
\hline
\end{tabular}

\subsection{Effects of nanotip geometries on the thermal evaporation}

Figure 9 shows the onset time of thermal evaporation of nanotips with different $R_{0}(1 \mathrm{~nm}, 3 \mathrm{~nm}$ and $5 \mathrm{~nm})$ and $H_{0}(80 \mathrm{~nm}$, $100 \mathrm{~nm}$ and $120 \mathrm{~nm}$ ) under various electric fields. It can be seen that the variation of $t_{e}$ with the electric field strength for different geometries shows similar four regions as discussed in the previous session. We find that those profiles can be fitted by using a cotangent relationship with the well-defined values of $E_{c}$ (the quantity that defines the minimum E-field strength required to initiate the thermal evaporation) and $E_{\max }$ (above this value, the thermal evaporation occurs immediately after the start of the MD simulation). Therefore, the following expression is employed in fitting

$$
t_{e}(E)=\tau \cot \left(\frac{\pi}{E_{\max }-E_{c}}\left(E-E_{c}\right)\right)+t_{h},
$$

where $\tau$ is a scaling parameter which has the same dimension as that of onset evaporation time and is an implicit function of both $R_{0}$ and $H_{0}$. The parameter $t_{h}$ is an average value of $t_{e}$ in the Region III as discussed in section 3.3.

The fitted parameters are listed in table 3 . Analyzing the variation of parameters under different conditions, it can be seen that fixing the radius of the tip hemisphere $\left(R_{0}\right)$, the increase of tip height only leads to the decrease of $E_{c}$, while there's no significant change in the overall trend of $t_{e}$. By fixing the total height of tips $\left(H_{0}\right)$ we see, that $\tau$ and $t_{h}$ grow linearly with $R_{0}$ and the range of the $t_{e}$ curve becomes wider as $R_{0}$ becomes larger. Thus in our simulations $R_{0}$ is the dominant factor which affects $t_{e}$. Therefore the properties such as electric field and electron emission current density at the apex region of the tip are mainly affected by $R_{0}$, which make thermal evaporation easier to occur in the ED-MD simulations.

From figure 9 and table 3, it is visible that $E_{c}$ decreases with increasing of the height of nanotips, while it increases with increasing of the radius of the tip hemisphere. In other words, the larger aspect ratio of nanotip gives smaller $E_{c}$ due to higher field enhancement factor and slower heat dissipation.

By calculating the maximum local electric field of the nanotips under the critical electric field $\left(E_{\text {apex }}=\gamma \cdot E_{c}\right)$, we find that the values of $E_{\text {apex }}$ are all around $12 \mathrm{GV} / \mathrm{m}$ regardless of the values of $R_{0}$ and $H_{0}$. Thus, a nonlinear relationship between $E_{c}$ and $\gamma$ is suggested, which can be obtained by fitting the calculated results to the following expression

$$
E_{c}=\frac{34.35}{\gamma^{1.526}}+0.19 \quad(\mathrm{GV} / \mathrm{m}) \text {. }
$$




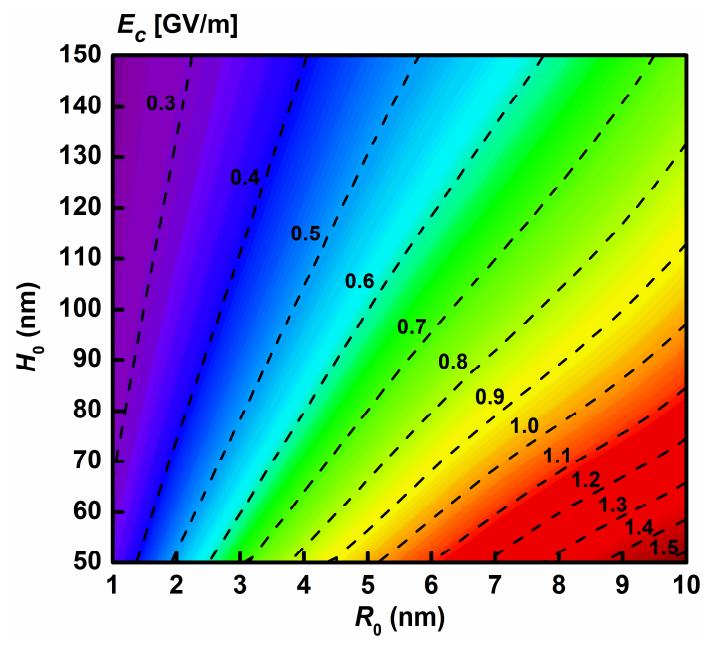

Figure 10. Prediction of $E_{c}$ using Eqs. (8) and (10) by varying $R_{0}$ from $1 \mathrm{~nm}$ to $10 \mathrm{~nm}$ and $H_{0}$ from $50 \mathrm{~nm}$ to $150 \mathrm{~nm}$

In order to quantitatively analyze the relationship between aspect ratio and $E_{c}$, we can convert aspect ratio into $\gamma$ by equation 8 and substitute the result into equations 10 . Thus, we can predict $E_{c}$ values for various nanotip geometries, i.e., varying the radius of the tip hemisphere $\left(R_{0}\right)$ from $1 \mathrm{~nm}$ to $5 \mathrm{~nm}$ and the total tip height $\left(H_{0}\right)$ from $75 \mathrm{~nm}$ to $150 \mathrm{~nm}$, as depicted in figure 10. The results show that the critical field strength for the initiation of thermal evaporation is ranged from $230 \mathrm{MV} / \mathrm{m}$ to $1.545 \mathrm{GV} / \mathrm{m}$. This is consistent with the experimentally determined vacuum breakdown field strength of $700 \mathrm{MV} / \mathrm{m}$ to $1.20 \mathrm{GV} / \mathrm{m}$ that was found in our previous work [43]. This suggests that field induced thermal evaporation down to the ps in time scale does play an important role in the initiation of the vacuum breakdown. However, defining the electric breakdown time as the onset time for the thermal runaway requires the further justification from both experiments and theoretical simulations.

We note that the sizes of nanotips or nano-electrodes used in experiments are still considerably larger than those of investigated in this work. The actual time scale of the onset thermal runway processes of experimental nano-electrodes (hundred nanometers long and several tens nanometers for tip radius) could be significantly longer than our predictions. Due to extremely high computational costs and poor parallel computing performance of ED-MD code, running the simulations for the tips, which would resemble those of experimental ones, is practically not doable. But our results imply that if we could continue the ED-MD simulations for the tips of bigger sizes, the onset thermal runaway time may gradually approach the experimental scale revealed by the discharge spectral images from glow discharge optical emission spectrometer. Specifically, the predicted onset time of thermal evaporation would gradually increase from several hundred picoseconds for our current nanotips to nano-seconds for experimental cases.
Furthermore, the local field on the tip apex is overestimated in our calculations because the electric field on the nanotip is obtained by solving the Laplace equation without considering the space charge effects. The presence of space charge substantially changes distribution of the electric field and current over the tips $[18,67]$. Consequently, the distribution of the E-field along the tip and the dynamics of its change with increasing temperature may also change, namely, the $\mathrm{E}$ decreases with increasing temperature. In this case, the maximum temperature is reached at a sufficiently large distance from the top [18]. Nevertheless, the electron emission and field distribution are calculated concurrently and consistently by GETELEC for each point on the surface in our model, since PIC simulations were not available at this stage, but the result could be considered good enough for the scope of this paper.

It is known that the space charge effect decreases the field enhancement factor $[18,38,67]$. Therefore, our calculations underestimate the onset time for the thermal evaporation and the corresponding critical electric field strength $E_{c}$. One possible solution to address this issue is to combine PIC method with ED-MD simulation, using $\gamma$ obtained from the former method as the input for the latter approach. New developments are underway to eventually address the above mentioned problems in the near future.

\section{Conclusions}

Aiming at the onset vacuum breakdown mechanism at nanoscale and the structural damages to nano-electrodes, we performed the state-of-the-art ED-MD simulations for nanotips under high electric field. It was found that the thermal runaway occurring in picosecond time-scale plays an important role for the initiation of the vacuum breakdown. By determining the onset time of atomic thermal evaporation and the critical electric field of nanoscale pre-breakdown for different nanotip geometries, it was shown that the onset time scale of atomic evaporation versus the critical field strength could be described by an empirical cotangent relationship. The aspect ratio of nanotips has a significant influence on the thermal evaporation of nanotips, which could be a way to predict the critical electric field of wider scale electrodes as a function of the height and the apex radius of the tip.

\section{Acknowledgements}

The work is partially supported by National Natural Science Foundation of China (51977169, 51607138, 51521065), Fundamental Research Funds for the Central Universities (sxzy022019069, xzy012019030), China Postdoctoral Science Foundation (2016M602820), A.K. and F.D. acknowledge the financial support of the CERN Kcontract (No. 47207461). We also acknowledge the Estonian scholarship program Kristjan Jaak, which is funded and 
managed by the Archimedes Foundation in collaboration with the Ministry of Education and Research of Estonia.

\section{References}

[1] M. Aicheler and CERN, CLIC Conceptual Design Report. Geneva: CERN, 2012

[2] Muranaka T, Blom T, Leifer K, et al. European Coordination for Accelerator Research and Development CON, 2011:22.

[3] Bajenescu T M I. Electrotehnica, Electronica, Automatica, 2014, 62(2):39-44

[4] Brown I G, Galvin J E and MacGill R A 1985 Appl. Phys.Lett. 47 358

[5] McCracken G 1980 J. Nucl. Mater. 933

[6] Dyke W, Trolan J, Martin E and Barbour J 1953 Phys. Rev.91 1043

[7] Dolan W, Dyke W and Trolan J 1953 Phys. Rev. 911054

[8] Williams D W , Williams W T . Journal of Physics D: Applied Physics, 1972, 5(2):280-290

[9] Williams D W , Williams W T . Journal of Physics D (Applied Physics), 1973, 6(6):734---743743

[10] Anders A, Anders S and Gundersen M A 1993 Phys. Rev. Lett.71 364

[11] Mesyats G 1993 JETP Lett. 5795

[12] Latham R V 1995 High Voltage Vacuum Insulation: Basic Concepts and Technological Practice (Amsterdam:Elsevier)

[13] Mesyats G A 1995 IEEE Trans. Plasma Sci. 23879

[14] Mesyats G A 2005 Plasma Phys. Control. Fusion 47 A109

[15] Descoeudres A, Levinsen Y, Calatroni S, Taborelli M and Wuensch W 2009 Phys. Rev. Spec. Top. 12092001

[16] Bugaev S P, Litvinov E A, Mesyats G A, et al. Soviet Physics Uspekhi, 1975, 18(1): 51

[17] Mesyats G A. High Power Microwave Generation and Applications. 1992, 10: 345

[18] Barengolts S A, Uimanov I V, Shmelev D L. IEEE Transactions on Plasma Science, 2019, 47(8): 3400-3405

[19] Barengolts S A, Oreshkin E V, Oreshkin V I, et al. IEEE Transactions on Plasma Science, 2019, 47(8): 3406-3411

[20] Barengolts S A, Mesyats V G, Oreshkin V I, et al. Physical Review Accelerators and Beams, 2018, 21(6): 061004

[21] Fu Y, Zhang P, Verboncoeur J P, et al. Plasma Research Express, 2020

[22] Go D B, Venkattraman A. Journal of Physics D: Applied Physics, 2014, 47(50): 503001

[23] Go D B. Journal of Physics D: Applied Physics, 2012, 46(3): 035202

[24] Fu Y, Zhang P, Krek J, et al. Applied Physics Letters, 2019, 114(1): 014102

[25] Fu Y, Krek J, Zhang P, et al. Plasma Sources Science and Technology, 2018, 27(9): 095014

[26] Meng G, Ying Q, Loveless A M, Wu F, Wang K, Fu Y, Garner A L and Cheng Y. Phys. Plasmas, 2019, 26014506

[27] Meng G, Gao X, Loveless A M, Dong C, Zhang D, Wang K, Zhu B, Cheng Y and Garner A L. Phys. Plasmas, 2018, 25 082116

[28] Meng G, Cheng Y, Gao X, et al. IEEE Transactions on Dielectrics and Electrical Insulation, 2018, 25(4): 1502-1507

[29] Loveless A M, Garner A L. Applied Physics Letters, 2016, 108(23): 234103
[30] Hirata Y, Ozaki K, Ikeda U, et al. Thin solid films, 2007, 515(9): 4247-4250

[31] Bhattacharjee S, Chowdhury T. Applied Physics Letters, 2009, 95(6): 061501

[32] Peschot A, Poulain C, Bonifaci N, et al. 61st IEEE Holm Conference on Electrical Contacts, San Diego, CA, 2015:280286

[33] Lyon D, Hubler A. 2013, IEEE Transactions on Dielectrics and Electrical Insulation, 20(4): 1467-1471

[34] Djurabekova F, Parviainen S, Pohjonen A and Nordlund K 2011 Phys. Rev. E 83026704

[35] Veske M, Parviainen S, Zadin V, et al. 2016, Journal of Physics D: Applied Physics, 49(21): 215301

[36] Veske M, Kyritsakis A, Eimre K, Zadin V, Aabloo A and Djurabekova F. 2017, J. Comp. Phys. 367279

[37] Kyritsakis A, Veske M, Djurabekova F, et al. 2017, Vacuum Nanoelectronics Conference. IEEE

[38] Kyritsakis A , Veske M , Eimre K , et al. 2018, Journal of Physics D: Applied Physics, 51(22):225203

[39] Meng G, Cheng Y, Dong C, et al. 2018 IEEE 2nd International Conference on Dielectrics (ICD). IEEE, 2018: 1-4

[40] Men C, Cheng Y, Zhu B, et al. 2016 International Conference On Condition Monitoring And Diagnosis (CMD). IEEE, 2016: 222-225

[41] Dong C, Cheng Y, Men C, et al. 2016 IEEE International Conference on Dielectrics (ICD). IEEE, 2016, 2: 1139-1142

[42] Meng G, Cheng Y, Dong C, et al. 2016 IEEE International Conference on Dielectrics (ICD). IEEE, 2016, 2: 1159-1162

[43] Meng G, Cheng Y, Wu K, et al. 2014, IEEE Transactions on Dielectrics and Electrical Insulation, 21(4): 1950-1956

[44] Schottky W 1923 Z. Phys. 1463

[45] Fowler R H and Nordheim L 1928 Proc. R. Soc. A 119173

[46] Murphy E L and Good R H 1956 Phys. Rev. 1021464

[47] Nottingham W B 1936 Phys. Rev. 4978

[48] Charbonnier F M, Strayer R W, Swanson L W and Martin E E, 1964 Phys. Rev. Lett. 13397

[49] K. Nordlund, Comput. Mater. Sci., vol. 3, pp. 448-456, 1994

[50] K. Nordlund, M. Ghaly, R. S. Averback, M. Caturla, T. D. de La Rubia, and J. Tarus, Phys. Rev. B, vol. 57, no. 13, p. 7556, 1998

[51] M. Ghaly, K. Nordlund, and R. S. Averback, 1999, Philos. Mag. A, 79(4) 795-820

[52] O.C. Zienkiewicz, R.L. Taylor, J.Z. Zhu, The Finite Element Method: Its Basis and Fundamentals, Seventh edition, Elsevier, Butterworth-Heinemann, Amsterdam, 2013

[53] Parviainen S, Djurabekova F, Timko H and Nordlund K 2011 Comput. Mater. Sci. 502075

[54] Eimre K, Parviainen S, Aabloo A, Djurabekova F and Zadin V 2015 J. Appl. Phys. 118033303

[55] Kyritsakis A and Xanthakis J P 2015 Proc. R. Soc. A 471 20140811

[56] Kyritsakis A and Xanthakis J 2016 J. Appl. Phys. 119045303

[57] Kyritsakis A and Djurabekova F 2017 Comput. Mater. Sci.128 15

[58] Bejan A and Kraus A D 2003 Heat Transfer Handbook vol 1 (New York: Wiley)

[59] Berendsen H J C, Postma J P M, van Gunsteren W F, DiNola A and Haak J R 1984 J. Chem. Phys. 813684

[60] Sabochick M J and Lam N Q 1991 Phys. Rev. B 435243 
[61] C. J. Edgcombe, U. Valdrè. Journal of Microscopy, 2001, [65] Roveri D S, Sant'Anna G M, Bertan H H, et al. Ultramicroscopy, 203(2):188-194

2015, 160:247-251

[62] Forbes R G, Edgcombe C J, U Valdrè. Ultramicroscopy, 2003, [66] Ester M, Kriegel H P, Sander J and Xu X 1996 Data Min. Knowl. 95(1-4): 57-65

[63] F.H. Read, N.J. Bowring. Nuclear Instruments \& Methods in Physics Research, 519(1-2):305-314

[64] G. C. Kokkorakis, A. Modinos, and J. P. Xanthakis, 2002, J. Discov. 96226

[67] Uimanov I V. IEEE Transactions on Dielectrics and Electrical Insulation, 2011, 18(3): 924-928

Appl. Phys., vol. 91, no. 7, p. 4580 